Annuaire suisse de politique de développement

26-2 | 2007

Financer le développement par la mobilisation des ressources locales

\title{
Le financement du développement : quelques portes d'entrée sur Internet
}

Lucas Oesch

\section{(2) OpenEdition \\ Journals}

Édition électronique

URL : http://journals.openedition.org/aspd/157

ISSN : 1663-9669

Éditeur

Institut de hautes études internationales et du développement

Édition imprimée

Date de publication : 1 novembre 2007

Pagination : 251-259

ISBN : 978-2-88247-068-3

ISSN : $1660-5934$

Référence électronique

Lucas Oesch, «Le financement du développement : quelques portes d'entrée sur Internet », Annuaire suisse de politique de développement [En ligne], 26-2 | 2007, mis en ligne le 24 juin 2009, consulté le 07 septembre 2020. URL : http://journals.openedition.org/aspd/157 


\title{
Le financement du développement: quelques portes d'entrée sur Internet
}

\author{
Lucas Oesch*
}

\section{Introduction}

En passant en revue un certain nombre de sites Internet portant sur le financement du développement, ce point d'appui vise à offrir au lecteur désireux d'approfondir ses connaissances sur le sujet une sélection de sites pertinents. Parmi les nombreux sites des organisations internationales, gouvernements nationaux, organisations non gouvernementales $(\mathrm{ONG})$ et centres de recherche qui portent sur la thématique du développement, ceux ayant un rapport avec son «financement» ont été sélectionnés, de manière non exhaustive.

Dans un premier temps, ce point d'appui présente tout d'abord les sites traitant du financement du développement parmi les organisations internationales, puis ceux de certains gouvernements nationaux européens et nord-américains (points 1 et 2). Dans un second temps (point 3) sont abordés les sites des ONG et des centres de recherche, avec tout d'abord une recension des sites portant de façon générale sur la thématique du financement du développement, suivie de la présentation de quelques sites d'ONG et de centres de recherche portant sur des thématiques plus spécifiques (microfinance, transferts des migrants, fiscalité, corruption, dette et ressources naturelles).

\section{Organisations internationales}

\section{Financing for Development Office, United Nations}

$<$ http://www.un.org/esa/ffd > (anglais)

Créé en 2002, à la suite de la Conférence internationale sur le financement du développement de Monterrey, ce secrétariat a pour but de faciliter la mise en place et le suivi des accords et des engagements formulés lors de la conférence. Son site, en langue anglaise, a comme objectif de mettre à disposition des informations sur le processus de suivi du «Consensus de Monterrey». On y trouve principalement les résolutions, rapports et autres publications des Nations unies portant sur le financement du développement, et ceci en plusieurs langues. Le site informe également des actions entreprises dans le cadre de la mise en place du «suivi intergouvernemental», ainsi que des diverses conférences et consultations qui se tiendront d'ici à la seconde moitié de 2008, lorsque se tiendra à Doha la nouvelle conférence sur le financement du développement. Il est à ce

* Doctorant à l'Institut universitaire d'études du développement (IUED), Genève. 
propos possible de s'inscrire à une newsletter mensuelle dénommée The Road to Doha. Ce site représente certainement la source incontournable de documents de première main pour toute personne s'intéressant au sujet, ainsi qu'une possibilité de se tenir au courant de l'actualité et des actions intergouvernementales entreprises dans le domaine.

Gender Responsive Budgeting, United Nations Development Fund for Women (UNIFEM)'

$<$ http://www.gender-budgets.org> (anglais, français et espagnol ${ }^{2}$ )

Inauguré en 2001, ce portail soutenu, entre autres, par le Fonds des Nations unies pour les femmes (UNIFEM) a fait peau neuve en 2007. Le but du site est de sensibiliser les acteurs du développement à l'approche genre dans les processus budgétaires. Un centre de documentation ( $>$ Resource Center) permet d'accéder à un grand nombre de documents de nature diverse (études, rapports...) en anglais, français, espagnol et arabe. On peut également accéder à de la documentation classée par régions (>Country Resources). Enfin, on trouve sur la page d'accueil du site (>Home) offre des communications actualisées de l'UNIFEM (>Message from UNIFEM).

\section{United Nations Conference on Trade and Development (UNCTAD)}

$<$ http://www.unctad.org > (anglais, français et espagnol) Home $>$ Programmes > International Investment Flows (World Investment Report)

La Conférence des Nations unies sur le commerce et le développement (CNUCED) publie chaque année un rapport présentant les tendances des investissements directs étrangers (IDE) dans le monde ainsi que des analyse de leurs incidences sur le développement. Le rapport complet n'existe qu'en anglais; des résumés (Aperçu) sont disponibles en plusieurs langues.

\section{Development Assistance Committee (DAC), Organisation for Economic Co-operation and Development (OECD)}

\section{$<$ http://www.oecd.org/dac> (anglais et français)}

Le Comité d'aide au développement (CAD) est l'organe de l'Organisation de coopération et de développement économiques (OCDE) chargé des questions de coopération au développement. Plusieurs départements de l'organisation ont affaire à la problématique du financement du développement. Le chemin d'accès et une description de la page du site jugée la plus pertinente sur le thème sont présentés ci-dessous. Les informations contenues sur le site du CAD sont essentielles car elles portent dans une large mesure sur les orientations générales des politiques de développement des pays «du Nord». Le site est en anglais; une version française, plus sommaire toutefois, existe également.

\footnotetext{
Je remercie Fenneke Reysoo pour ses indications fournies sur ce site.

2 Ici et dans tout le texte, lorsqu'un site existe en plusieurs langues, la première langue citée est la langue principale du site.
} 
Home $>$ Related Topics $>$ Development $>$ Finance for Development

Maintenu par le Centre de développement de l'OCDE, un organe qui collabore étroitement avec le $\mathrm{CAD}$, ce site met principalement à disposition des études, documents de travail, documents de repère et des synthèses (Policy Insights et Policy Briefs) portant sur des sujets rattachés au financement du développement. Il est possible de les obtenir en vrac (>Publications \& Documents) ou classés par pays (>Information by Country). La page de publications et de documents propose également un lien vers une nouvelle publication annuelle lancée en 2007, le rapport Financing Development: Aid and Beyond (>Annual Reports), qui n'est cependant pas encore accessible dans son intégralité. Enfin, le site propose un lien vers un forum (>OECD Global Forum on Development), dont le thème pour la période 2006-2009 est Financing Development Effectively. Les comptes rendus et programmes des diverses rencontres proposées dans le cadre du forum y sont disponibles.

Home $>$ Related Topics $>$ Development $>$ Aid Effectiveness et/ou Investment for Development

Il s'agit de deux autres chemins d'accès abordant la problématique du financement du développement.

\section{International Monetary Fund (IMF)}

$<$ http://www.imf.org > (anglais, français et espagnol), Home >Publications $>$ Series Titles: Finance and Development $(F \& D)$

Finance and Development est un magazine trimestriel publié en anglais, en français et en espagnol par le Fonds monétaire international (FMI), et qui porte sur divers sujets liés à des questions de finance, de développement, ainsi que de financement du développement. Les archives (depuis 1996) ainsi que le numéro le plus récent y sont accessibles.

\section{World Bank}

$<$ http://www.worldbank.org > (anglais, français et espagnol), Home $>$ Data \& Research $>$ Prospects $>$ Publications $>$ Global Development Finance

Le rapport annuel Global Development Finance, publié par la Banque mondiale, porte sur «le financement du développement dans le monde» et traite chaque année d'un nouveau thème s'y rattachant. Il se propose principalement d'établir un diagnostic des flux récents de capitaux en direction des pays en développement et d'imaginer leurs perspectives futures. Le rapport en anglais est disponible depuis l'édition de 1999, ainsi que des résumés dans d'autres langues. 


\section{Gouvernements}

\section{Union européenne}

$<$ http://europa.eu> (langues européennes)

$\square$ EuropeAid Co-operation Office

$<$ http://ec.europa.eu/europeaid >

Le site permet d'accéder à des activités de microfinance soutenues par l'agence (>Programmes and Projects $>\mathrm{ACP} / \mathrm{EU}$ Microfinance). La page de documentation de l'agence (>Reports and Publications) contient également une série de documents intéressants sur les différentes méthodes d'allocation de l'aide (Aid Delivery Methods): aide par projet, aide budgétaire...

\section{Suisse}

<http://www.admin.ch> (anglais, français, allemand et italien)

Au sein de l'administration fédérale, deux entités en particulier s'occupent de questions liées au financement du développement:

$\square$ Direction du développement et de la coopération (DDC)

$<$ http://www.ddc.admin.ch>Accueil $>$ Thèmes $>$ Economie et emploi

$>$ Développement du secteur financier

Cette page présente le travail de la Direction du développement et de la coopération (DDC) en matière de promotion de l'accès des populations des pays en développement aux services financiers (épargne, crédit, transferts d'argent et assurances). On y trouve également de la documentation relative aux différents services financiers.

$\square$ Centre de prestations Coopération et développement économiques

(Secrétariat d'Etat à l'économie, SECO)

$<$ http://www.seco-cooperation.admin.ch> Accueil > Thèmes

$>$ Soutien macroéconomique

Cette page est consacrée aux activités du Secrétariat d'Etat à l'économie (SECO) en matière d'aides budgétaires et d'aide à la mise en place de secteurs financiers et bancaires dans les pays en développement. Sont en outre présentées les mesures de désendettement soutenues par la Confédération helvétique.

\section{France}

$\square$ Ministère des affaires étrangères et européennes

$<$ http://www.diplomatie.gouv.fr> (français, anglais, allemand et espagnol), Accueil $>$ Actions de la France $>$ Financements innovants du développement 


\section{Allemagne}

$\square$ Deutsche Gesellschaft für Technische Zusammenarbeit (GTZ)

$<$ http://www.gtz.de> (allemand et anglais)

Startseite $>$ GTZ - Themen $>$ Wirtschaft und Beschäftigung $>$ Finanzsysteme

Startseite >GTZ - Themen > Good Governance >Öffentliche Finanzen et/ou Korruption

\section{Royaume-Uni}

$\square$ Department for International Development (DFID)

$<$ http://www.dfid.gov.uk> (anglais), Home > Millennium Dev't Goals > Aid, Trade, Growth \& Global Partnership >Aid effectiveness

\section{Etats-Unis}

$\square$ United States Agency for International Development (USAID)

$<$ http://www.usaid.gov> (anglais) Home >Our Work > Economic Growth \& Trade

\section{Canada}

$\square$ Canadian International Development Agency (CIDA)

$<$ http://www.acdi-cida.gc.ca> (anglais et français) Home > Publications

$>$ Policy Suite > Policy Statement on Strengthening Aid Effectiveness

\section{Organisations non gouvernementales (ONG) et centres de recherche}

\section{Eldis}

$<$ http://www.eldis.org > (anglais)

Il s'agit d'un portail, géré par l'Institute of Development Studies (IDS) et financé par diverses agences nationales de coopération. L'option de recherche (>Search Eldis) permet de rechercher divers documents émanant d'organisations actives dans le développement. Il est possible d'utiliser les mots clés «financing development ».

\section{Europe's Forum on International Cooperation (EUFORIC)}

$<$ http://www.euforic.org > (anglais et français)

Ce Forum européen de coopération internationale est soutenu par diverses agences nationales de coopération, $\mathrm{ONG}$ et instituts de recherche sur le développement. Le page de recherche (>Search) permet d'accéder à des documents et à d'autres sites Internet portant sur le développement, à travers quatre moteurs de 
recherche différents. Les mots clés «financing development» débouchent sur des résultats intéressants dans ces quatre moteurs.

\section{World Institute for Development Economics Research, United Nations University (UNU-WIDER)}

$<$ ttp://www.wider.unu.edu> (anglais)

La page de recherche (>Search) de ce site permet d'accéder aux travaux de recherche et de discussion en anglais, produits par les chercheurs rattachés à l'institut. Les mots clés «finance» ou «financing » conduisent à des documents intéressants portant sur le financement du développement.

\section{Deutsches Institut für Entwicklungspolitik (DIE)}

$<$ http://www.die-gdi.de> (allemand, anglais, français et espagnol) Home $>$ Research and Consultancy >Focal area IV: Dept. V: World Economy and Development Financing

Le site présente le département et ses différents projets de recherche concernant les questions d'économie et de financement du développement.

\section{World Economic Forum (WEF)}

$<$ http://www.weforum.org > (anglais) Home > Initiatives

$>$ Financing for Development

Le site met en avant les partenariats public-privé (PPP) et les investissements privés comme outils de développement potentiels.

\section{Gerster Consulting (Gerster Development Consultants)}

$<$ http://www.gersterconsulting.ch > (anglais) Home $>$ Results $>$ Finance

Le site donne accès aux travaux réalisés par le cabinet de consultants sur les questions de financement. On y trouve un document intitulé New Sources of Development Financing: An SDC Working Paper, produit en 2005 pour la Direction du développement et de la coopération (DDC) de l'administration fédérale.

\section{Global Policy Forum (GPF)}

$<$ http://www.globalpolicy.org> (anglais) Home >Social \& Economic Policy $>$ Financing for Development

Cette ONG se propose de suivre et d'analyser les décisions et les politiques adoptées au sein des Nations unies, et ceci notamment en matière de financement du développement. 


\section{Choike (Third World Institute, ITEM)}

$<$ http://www.choike.org> (anglais et espagnol) Home >In-depth Reports

$>$ Society: Financing for Development

Il s'agit d'un portail géré par l'ONG Third World Institute (ITEM). La page consacrée au financement du développement propose notamment une série de liens intéressants (Related links).

\section{Coordination Sud}

$<$ http://www.coordinationsud.org > (français) Accueil

$>$ Positions des $O N G>$ Financement $d u$ dvlpt

Ce portail des ONG françaises actives dans la solidarité internationale permet notamment de se tenir au courant de l'actualité des questions de financement du développement.

\section{European NGO Confederation for Relief and Development (CONCORD)}

$<$ http://www.concordeurope.org > (anglais et français) Home >Topics

$>$ Financing for development - Aidwatch

Ce réseau d'ONG européennes propose notamment un suivi et une analyse de la quantité et de la qualité de l'aide publique au développement (APD) allouée par l'Union européenne et ses Etats membres.

\section{- Microfinance}

\section{Portail microfinance}

$<$ http://www.lamicrofinance.org > (français et anglais)

Soutenu par diverses agences nationales de coopération, des ONG et des instituts de recherche, ce portail offre une quantité importante de documentation sur différents thèmes liés à la microfinance (>Dossiers thématiques). Une recherche par mots clés est également possible (>Bibliothèque). Enfin, une rubrique actualisée de brèves (>Actualités) permet de se tenir au courant de l'évolution du sujet et une page de liens (Autres ressources $>$ Liens) contient des descriptions concises d'autres sites Internet traitant de la microfinance.

\section{Forum finances et développement (Epargne sans frontière, ESF)}

$<$ http://esf.asso.fr> (français et anglais)

Maintenu par l'association Epargne sans frontière, ce portail permet, comme le précédent, de s'informer sur divers thèmes liées à la microfinance, à partir de sa page d'accueil (>Accueil du site). De plus, il donne accès à la revue Techniques financières et développement, publiée par l'association (>La revue: Techniques financières et développement). Il est également possible de participer à des forums de discussion ( $>$ Nos forums). 


\section{Transferts des migrants}

\section{Remittances.eu \\ (Foundation for International Migration and Development, FIMD)}

$<$ http://www.remittances.eu> (anglais)

Sur sa page principale (>Home), le site de la fondation donne accès à des brèves et à diverses publications récentes (Newest publications) portant sur le thème des transferts des migrants. Il est également possible de consulter les exemplaires d'une newsletter intitulée Migrant Remittances.

\section{- Fiscalité}

\section{Tax Justice Network}

<http://www.taxjustice.net/cms> (anglais, français, allemand et espagnol)

Des explications sur différents thèmes liés à la fiscalité (fraude fiscale, compétition fiscale...) sont présentées sur le site de cette ONG (>Resources). Au moment de la rédaction de ce point d'appui, de nombreuses rubriques étaient cependant encore en construction. Dans sa rubrique de brèves (News), des liens actualisés vers des articles de revues ou de presse, ainsi que des rapports et des annonces de conférences, sont disponibles.

\section{Interportal.ch}

$<$ http://www.interportal.ch> (anglais, français et allemand) Home >Contenu $>$ Dossiers $>$ Evasion fiscale, concurrence fiscale et pays en développement

Financé par des ONG suisses, ce portail permet de se tenir au courant sur de nombreux thèmes ayant affaire au développement ainsi qu'à la coopération internationale, dont notamment, en ce qui concerne le financement du développement, les questions de fiscalité.

\section{- Corruption}

\section{Transparency International}

$<$ http://www.transparency.org > (anglais)

Le site documente les thèmes de travail de l'ONG (>Global priorities) et met à disposition une sélection de ses publications (>Publications) ainsi que des guides d'analyse et de lutte contre la corruption (>Policy and research). Il offre également une page actualisée de communiqués de presse ( $>$ News room) publiés par l'organisation et ses diverses agences régionales. 


\section{European Network on Debt and Development (EURODAD)}

$<$ http://www.eurodad.org > (anglais, français et espagnol)

Le site de ce réseau d'ONG européennes présente, au travers de deux rubriques différentes (Debt/Aid), une description de différents thèmes liés à l'aide au développement et à la question de la dette (différents types de dettes, efficacité de l'aide...). Des rapports et articles d'actualité sont disponibles pour chaque thème.

\section{Comité pour l'annulation de la dette du tiers-monde (CADTM)}

$<$ http://www.cadtm.org > (anglais, français et espagnol)

Le site de ce comité propose également un grand nombre d'informations sur les questions liées à la dette (chiffres, actualités...).

\section{- Ressources naturelles}

\section{The Extractive Industries Transparency Initiative (EITI)}

$<$ http://www.eitransparency.org > (anglais)

Le site de l'organisation, financée en partie par la Banque mondiale et divers gouvernements nationaux, propose une revue de la gouvernance des ressources naturelles (pétrole, gaz...) dans les pays qui en sont richement dotés (>EITI Countries). Une page bibliographique du site (>Publications) recense également un certain nombre de rapports et d'ouvrages liés à la thématique. Enfin, le site contient une page actualisée d'articles de presse (>EITI News)

\section{Publish What You Pay coalition}

$<$ http://www.publishwhatyoupay.org > (anglais, français, allemand, espagnol et autres)

Le site de cette coalition d'ONG, militant pour une plus grande transparence dans les paiements des compagnies extractives et dans les revenus des gouvernements, constitue également une source d'information sur le sujet. 